\title{
Knee osteoarthritis at the early stage: The four-week effect of lateral wedge insole on pain and risk of falls
}

\author{
Elham Esfandiari ${ }^{1}$, Mohammad Ali Sanjari ${ }^{2 *}$, Ali Ashraf Jamshidi ${ }^{3}$, Mojtaba Kamyab ${ }^{1}$, \\ Hamid Reza Yazdi ${ }^{4}$
}

Received: 21 Apr 2017

Published: 3 Mar 2018

\begin{abstract}
Background: Knee osteoarthritis (KOA) is associated with a decrease in function, increase in pain and risk of falls. Lateral wedge insole (LWI) is commonly prescribed in KOA to improve pain and function. Our study aimed to 1) evaluate the clinical symptoms and risk of falls in early KOA and compare with controls; 2) evaluate the immediate and four-week effect of LWI.

Methods: A sample of 20 Persian dwelling individuals with early KOA and 19 matched controls were recruited. Pain with Visual Analogue Scale (VAS), Quality of life (QOL) with the knee injury and osteoarthritis outcome score, risk of falls with the Timed Up and Go (TUG) and static One-leg Balance (OLB) tests were assessed. The four-week effect of $5^{\circ}$ LWI was considered for individuals with KOA. Independent t-test was done to report the between-group differences, and paired t-test was used to report the four-week effect of LWI.

Results: At baseline, statistically significant higher scores for pain, lower scores for QOL, and higher risk of falls were observed in KOA compared to controls $(\mathrm{p}<0.001)$. A significant statistical decrease was observed in pain, and risk of falls, and an increase in QOL in KOA after four-week effect of LWI compared to baseline $(p<0.001)$.

Conclusion: People with early KOA showed higher pain and lower level of QOL that were associated with higher risk of falls. LWI may have the potential to improve clinical symptoms and reduce the risk of falls at the early stage of KOA.
\end{abstract}

Keywords: Osteoarthritis, Knee, Symptoms, Accidental falls, Foot orthoses

Copyright $@$ Iran University of Medical Sciences

Cite this article as: Esfandiari E, Sanjari MA, Jamshidi AA, Kamyab M, Yazdi HR. Knee osteoarthritis at the early stage: The four-week effect of lateral wedge insole on pain and risk of falls. Med J Islam Repub Iran. 2018(3 Mar);32:17. https://doi.org/10.14196/mjiri.32.17

\section{Introduction}

Osteoarthritis is a common degenerative joint disease associated with chronic pain and functional limitation in older adults (1). The prevalence of symptomatic knee osteoarthritis $(\mathrm{KOA})$ was reported as $17 \%$ in people aged $\geq$ 45 years (2). KOA has the $11^{\text {th }}$ highest global rank of disability among 291 conditions (3). In addition, age and obesity are considered as two important systemic risk factors in the incidence of KOA (4); therefore, older adults are at higher risk of KOA due to aging and increasing obesity in world's population (3).

KOA initiates changes in knee joint cartilage, ligaments,

Corresponding author: Dr Mohammad Ali Sanjari, sanjarima@alum.sharif.edu

1. Department of Orthotics and Prosthetics, School of Rehabilitation Sciences, Iran University of Medical Sciences, Tehran, Iran.

2. Department of Basic Rehabilitation Sciences, School of Rehabilitation Sciences, Iran University of Medical Sciences, Tehran, Iran.

3. Department of Physical Therapy, School of Rehabilitation Sciences, Iran University of Medical Sciences, Tehran, Iran.

4. Department of Orthopaedic Surgery, School of Medicine, Iran University of Medical Sciences, Tehran, Iran. tendons and muscles (5), which lead to knee buckling, psychosocial outcomes, fear of falling, balance deficits, and limitation in certain physical activities (6-8). Both clinical status and functional disability lead to a decrease in quality of life in individuals with KOA (1).

Variety of interventions are recommended for managing symptoms and reducing the speed of disease progression (9). While arthroplasty and surgical treatments are chosen as the therapeutic option for the late stage of the disease (10); Non-pharmacological and conservative treatments are at the first line of early KOA management (9). Lateral

$\uparrow$ What is "already known” in this topic:

People with KOA suffer from pain, increased risk of falls, and decreased quality of life. To date, most studies focused on later stage of KOA. Moreover, most conservative treatments, e.g. shoe modifications and orthoses, showed controversial effects on clinical symptoms in KOA.

\section{$\rightarrow$ What this article adds:}

Our study outlined a difference in clinical symptoms and a higher risk of falls in people with early KOA. LWI for four weeks is associated with improvement in clinical symptoms and decreasing risk of falls at the early stage of KOA. 
wedge insole (LWI) is a conservative and inexpensive treatment that is widely prescribed for improvement of pain, symptoms and decreasing knee medial compartment loading $(11,12)$. However, there is a controversy on clinical effects of LWIs in KOA in different studies $(13,14)$. However, KOA is growing in general population and there is lack of evidence about early KOA. Therefore, further investigation is needed to clarify previously reported clinical symptoms and adaptations in established earlier stages of KOA; since the possibility of decreasing progression speed of the early cartilage defects, particularly in younger people, has been reported. (15) Therefore, it is extremely important to reverse the disease progression using early conservative interventions.

The objective of this study was to 1) evaluate pain, quality of life, and risk of falls in early KOA; 2) evaluate the four-week effect of LWI on pain, quality of life, and risk of falls in early KOA. We hypothesized that: individuals with early KOA had higher scores in pain and lower scores in quality of life. Moreover, the individuals with early KOA suffered from a higher risk of falls. We also hypothesized that LWI decreases pain associated with an increase in quality of life and a decrease in risk of falls in early KOA.

\section{Methods \\ Patients \\ Study design and patient recruitment}

This quasi-experimental study was approved by the ethics committee of Iran University of Medical Sciences. All subjects signed written informed consent form.

KOA group: Twenty individuals with evidence of early KOA were recruited to this study. The early KOA was diagnosed and confirmed using radiographs and MRI scans by consultant radiologists and orthopaedic surgeons $(16,17)$.

Inclusion criteria were: early KOA in at least one knee, being older than 35 years old, having pain greater than 3 according to visual analogue scale (VAS) in the medial aspect of the knee, mild medial joint space narrowing which was defined as the $15-25 \%$ of the joint space narrowing in the medial compartment, and knee stiffness. Exclusion criteria were secondary osteoarthritis, neurological disorders, rheumatoid or other inflammatory arthritis, any musculoskeletal conditions, age younger than 35 or greater than 65 years old, Body mass index (BMI) greater than $30 \mathrm{~kg} / \mathrm{m}^{2}$, back pain, knee surgery, foot problems that prohibited using insoles, concomitant physical therapy, and inability to walking independent.

Control group (CTL): Nineteen age- and sex-matched healthy individuals were recruited in the control group from staffs and students of hospital, faculty, and gym. They all had no clinical evidence of KOA or neuromusculoskeletal pathology.

\section{General demographic evaluation}

A self-designed demographic form was filled for all participants. The demographic form included data regarding age, sex, body weight and height. The BMI was calculated for each participant.

\section{Interventions}

The analysis was conducted in the context of three conditions, including barefoot, individual's shoes, and LWI put in individual's shoes. LWI consisted of a 5-degree wedge, from high-density ethyl vinyl acetate, in lateral border of the foot which was posted just proximal to the fifth metatarsal head (Fig. 1). The wedge of 5 degrees was chosen as it was shown it is more comfortable for individuals with KOA rather than higher wedges (18). Neutral insole had no lateral wedge for uninvolved limb. The insoles were constructed from low-density ethyl vinyl acetate for shock absorbing. The insoles were fitted in the own shoes of each individual under the shoe liner. The examiner considered the individuals' shoes to be appropriate for the use of LWIs. There was a mild medial longitudinal arch support embedded in each insole for individual's foot comfort (Fig. 2). At baseline, for adaptation to insoles, individuals wore the LWIs in their own shoes for 20 minutes. For evaluation of four-week effect, the individuals with KOA were instructed to use the LWI in an appropriate shoe without a high heel and narrow toe box, for 5 to 10 hours a day (19). Because the insoles can biomechanically be effective in reducing medial compartment knee loading (18), the four-week effect of the insoles was considered only for individuals with KOA.

\section{Outcome measures}

An examiner assessed all individuals at baseline and

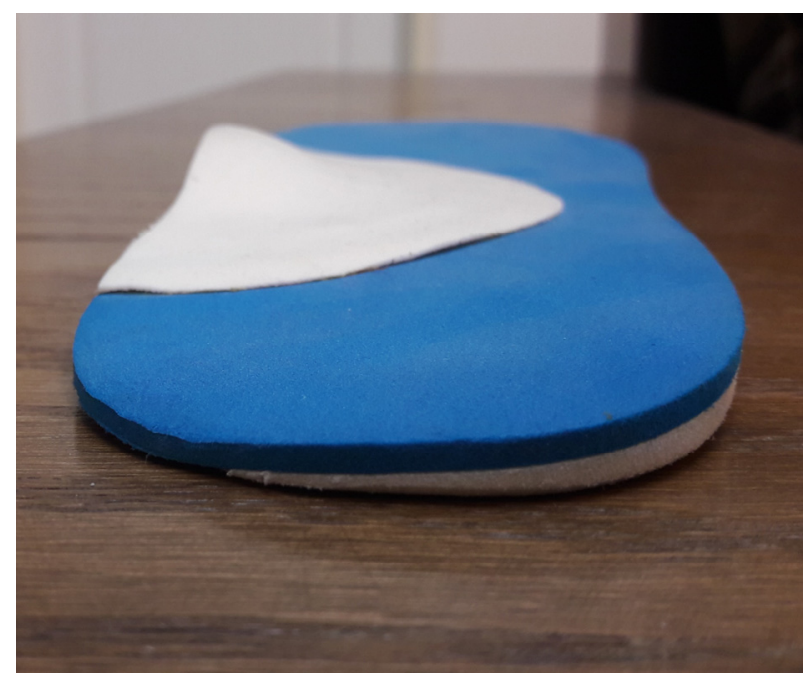

Fig. 1. Lateral Wedge Insole for the Right Foot

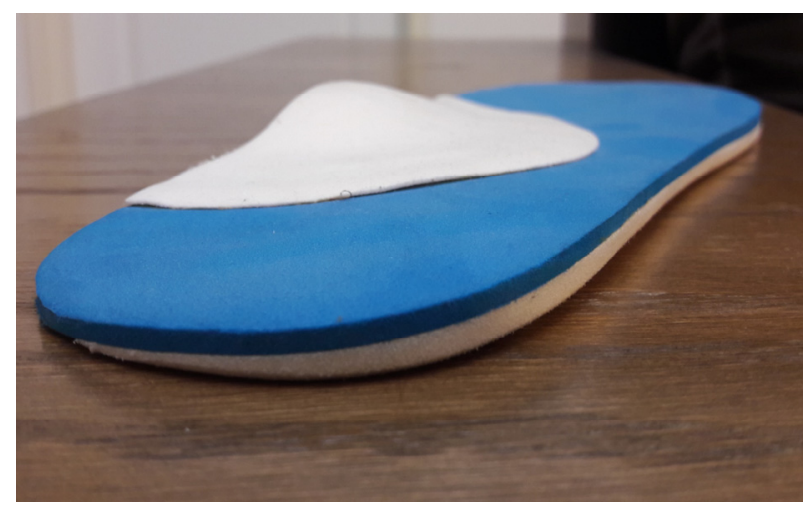

Fig. 2. The lateral wedge insole with mild medial longitudinal arch support 
KOA group at four-week follow up. Baseline demographic information was collected. In addition, individuals' expectations about the beneficial effect of the treatment with LWI were collected (13). A 5-point ordinal scale was used $(1=$ no effect, $5=$ complete recovery). Higher scores indicated higher expectations (13).

\section{Symptomatic measures}

Primary outcome measure: Average knee pain in the past week was assessed using $100 \mathrm{~mm}$ VAS. Knee pain is a well-accepted clinimetric property for the clinical trials in $\operatorname{KOA}(20)$.

Secondary outcome measures: Pain on walking was measured using $100 \mathrm{~mm}$ VAS. Knee related quality of life was also measured using Persian version of Knee Injury and Osteoarthritis Outcome Score (KOOS) (21). In addition, global change in pain and physical function perceived by individuals compared with baseline, were recorded by a 5-point ordinal scale (including much better, slightly better, no change, slightly worse and much worse).

The measurement was dichotomized into improvement (slightly better and much better) and no improvement (much worse, slightly worse, and no change). In addition, individuals in KOA group recorded daily use and discomfort of their insoles. The comfort of the insole was recorded by a $100 \mathrm{~mm}$ VAS, as the 0 was extremely uncomfortable and 100 was extremely comfortable. The individuals ranked the comfort of their insoles at baseline and after four-week use.

\section{Risk of falling}

All individuals were interviewed in regards to fall experience in the previous year by asking the following question: "Have you experienced falls during the past 12 months" and also "How many times did you have the experience of falls?" $(8,22)$. A fall was defined as a sudden and unintentional change in position of a person to rest on a lower level like floor, ground, or other objects (23). Fall risk was assessed using two validate clinical tools, the Timed Up and Go (TUG) test (24), and simple clinical static One-leg Balance (OLB) test (25). In TUG, individuals were instructed to get up from a standard armchair chair (height of seat was $48 \mathrm{~cm}$, and arms $68 \mathrm{~cm}$ ) without the assistant of their hands use, walk for $3 \mathrm{~m}$, turn around the placed obstacle at a 3 meter distance from the chair, and return to a fully seated position on the chair (24). Three trials were recorded for each individual in each condition. The time of this test was recorded for each individual by a stop watch.

In OLB test, the time of performance was recorded in seconds by a stop watch as the person lifted one foot from the ground to the time the balance lost and again foot touched the floor (maximum 60 seconds for each foot). The longer time indicated the better balance ability. Moreover, any observation of change in the position of arms spacing during the first 5 seconds of the performance was recorded as dichotomously, yes or no (25). This test was performed for both legs for three conditions.

All symptomatic measures and risk of falling variables were measured at baseline for KOA group and CTL, and four-week follow-up for individuals with KOA in three conditions, including barefoot, individual's own shoes, and LWI put in individual's own shoes, in random order.

\section{Statistical analysis}

All statistical analysis was conducted by SPSS v. 22. A p-value less than 0.05 was considered as the statistically significant. For continuous variables paired t-test was used to compare the differences in mean change of measurements (follow-up compared to baseline). In addition, independent sample t-test was conducted to compare mean differences of continuous variables among KOA group (at baseline and four-week follow-up) and CTL. To compare nominal variables between two groups, analysis of Chi square was applied. Also, repeated measurement ANOVA was used to assess the difference between three conditions.

\section{Results}

\section{General Demographic}

Twenty individuals with KOA and 19 CTL individuals participated in this study. The majority of individuals were female (90\% in KOA group and $89 \%$ in CTL). The mean ages of KOA group and CTL group $(\mathrm{M}=52.95, \mathrm{SD}=7.15$; and $\mathrm{M}=47.47, \mathrm{SD}=11.23$ years, respectively) were not significantly different $(\mathrm{t}(37)=-1.98, \mathrm{p}=0.06)$. In addition, the mean BMIs of KOA group and CTL group ( $\mathrm{M}=26.86$, $\mathrm{SD}=1.49 ;$ and $\mathrm{M}=25.89, \mathrm{SD}=1.80 \mathrm{~kg} / \mathrm{m}^{2}$, respectively) were not statistically different $(\mathrm{t}(37)=-1.91, \mathrm{p}=0.06)$.

\section{Symptoms}

At baseline, the KOA group had significantly higher scores in average knee pain and pain in walking during last week $(\mathrm{t}(37)=10.59$, and 5.12; $\mathrm{p}<0.001$, respectively). In addition, lower score in knee related QOL subscale of $\operatorname{KOOS}(\mathrm{t}(37)=13.66 ; \mathrm{p}<0.001)$ was observed in KOA group compared to CTL group. But there was no significant difference in shoes comfort with LWI between KOA group at baseline and CTL group $(\mathrm{t}(37)=-1.75 ; \mathrm{p}=0.09)$. Table 1 showed the descriptive result of clinical symptoms in CTL, KOA group at baseline and KOA group at four-week follow-up.

\section{Risk of falling}

At baseline, there was an obvious difference in the experience of falls in the last 1 -year between KOA group at baseline and CTL group $\left(\mathrm{X}^{2}(2, \mathrm{~N}=56)=6.37 ; \mathrm{p}=0.04\right)$, with an average number of 2.18 and .22 in the KOA and CTL respectively. Moreover, there was a significant difference in time of OLB test of both right and left limb in three barefoot, LWI and shoes conditions between KOA group at baseline and CTL group $(\mathrm{p}<0.001)$ (Table 2). Also, a significant difference was observed in time of TUG test in barefoot and LWI conditions between KOA group at baseline and CTL group. Table 2 shows the detail of risk of falls in both groups. 
Table 1. Characteristics of participants by group, including control (CTL), knee osteoarthritis group (KOA) at baseline, and knee osteoarthritis group at four-week follow-up

\begin{tabular}{|c|c|c|c|}
\hline Variables & $\begin{array}{l}\text { CTL Group (19) } \\
\text { Mean } \pm \text { SD }\end{array}$ & $\begin{array}{c}\text { KOA Group at baseline (20) } \\
\text { Mean } \pm \text { SD }\end{array}$ & $\begin{array}{l}\text { KOA Group at four-week follow up (20) } \\
\text { Mean } \pm \text { SD }\end{array}$ \\
\hline average knee pain in the past week $(0-100)$ & $3.68 \pm 5.97$ & $66.50 \pm 25.18$ & $30.00 \pm 19.19$ \\
\hline $\begin{array}{l}\text { pain on walking }(\mathrm{o}-100) \\
\text { KOOS}^{*}\end{array}$ & $0.00 \pm 0.00$ & $71.00 \pm 24.89$ & $23.00 \pm 26.77$ \\
\hline knee related $\mathrm{QOL}^{\dagger}(0-100)$ & $92.11 \pm 14.85$ & $25.31 \pm 15.63$ & $46.56 \pm 24.12$ \\
\hline comfort with shoes with lateral wedge insole $(0-100)$ & $95.26 \pm 7.72$ & $88.00 \pm 16.41$ & $96.50 \pm 6.71$ \\
\hline $\mathrm{TUG}^{+}-\mathrm{WO}^{\mathrm{s}}(\mathrm{ms})$ & $8.31 \pm 0.94$ & $11.72 \pm 3.08$ & $9.77 \pm 2.24$ \\
\hline TUG-WS ${ }^{* *}(\mathrm{~ms})$ & $9.50 \pm 5.97$ & $12.04 \pm 3.16$ & $9.87 \pm 2.03$ \\
\hline right $\mathrm{OLB}^{+\star}-\mathrm{WO}(\mathrm{ms})$ & $51.82 \pm 10.55$ & $26.20 \pm 20.57$ & $31.20 \pm 19.90$ \\
\hline right OLB-WS (ms) & $50.88 \pm 10.48$ & $18.50 \pm 17.53$ & $30.39 \pm 19.95$ \\
\hline right OLB-WI (ms) & $53.41 \pm 9.51$ & $27.02 \pm 20.48$ & $33.17 \pm 18.90$ \\
\hline left OLB-WO (ms) & $47.04 \pm 12.48$ & $25.88 \pm 19.19$ & $30.09 \pm 17.52$ \\
\hline left OLB-WS (ms) & $53.96 \pm 10.87$ & $19.60 \pm 17.08$ & $31.34 \pm 16.20$ \\
\hline left OLB-WI (ms) & $53.29 \pm 10.59$ & $22.72 \pm 16.65$ & $32.37 \pm 18.60$ \\
\hline
\end{tabular}

left OLB-WI (ms) $53.29 \pm 10.59$

* Knee Osteoarthritis Outcome Score

${ }^{\dagger}$ Quality of Life

\$ Timed Up and Go Test

s Barefoot

${ }^{* *}$ With Shoes

${ }^{++}$With Lateral Wedge Insoles

林 Static One-leg Balance Test

Table 2. Frequency of Arm movement in one leg balance test (OLB) and experience of falls in control (CTL) subjects and individuals with knee osteoarthritis (KOA) at baseline and four-week follow-up

\begin{tabular}{|c|c|c|c|c|c|c|}
\hline \multirow[t]{2}{*}{ Variables } & \multirow[t]{2}{*}{$\begin{array}{l}\text { CTL Group } \\
\text { (19) }\end{array}$} & \multirow[t]{2}{*}{$\begin{array}{c}\text { KOA Group at } \\
\text { baseline }(20)\end{array}$} & \multirow[t]{2}{*}{$\begin{array}{l}\text { KOA Group at four- } \\
\text { week follow up (20) }\end{array}$} & \multicolumn{2}{|c|}{$\begin{array}{l}\text { P-value of frequency difference } \\
\text { between groups }\end{array}$} & \multirow{2}{*}{$\begin{array}{c}\text { P-value of frequency } \\
\text { difference within groups } \\
\text { KOA at baseline-KOA at } \\
\text { four-week follow-up }\end{array}$} \\
\hline & & & & $\begin{array}{l}\text { CTL-KOA at } \\
\text { baseline }\end{array}$ & $\begin{array}{l}\text { CTL-KOA at four- } \\
\text { week follow-up }\end{array}$ & \\
\hline Right OLB-WO & $5(25.31 \%)$ & $6(30 \%)$ & $3(15 \%)$ & 0.51 & 0.78 & 0.22 \\
\hline Right OLB-WS= & $3(15.79 \%)$ & $6(30 \%)$ & $4(20 \%)$ & 0.54 & 0.07 & $0.03 *$ \\
\hline Right OLB-WI & $-0-$ & $3(15 \%)$ & $-0-$ & $\mathrm{N} / \mathrm{A}^{\#}$ & $\mathrm{~N} / \mathrm{A}^{\#}$ & $\mathrm{~N} / \mathrm{A}^{\#}$ \\
\hline Left OLB-WO & $2(10.53 \%)$ & $5(25 \%)$ & $3(15 \%)$ & 0.46 & 0.18 & 0.07 \\
\hline Left OLB-WS & $1(5.26 \%)$ & $6(30 \%)$ & $-0-$ & $<0.001^{*}$ & $\mathrm{~N} / \mathrm{A}^{\dagger}$ & $\mathrm{N} / \mathrm{A}^{\dagger}$ \\
\hline Left OLB-WI & $1(5.26 \%)$ & $2(10 \%)$ & $1(5 \%)$ & 0.78 & 0.68 & 0.73 \\
\hline \multicolumn{7}{|c|}{ Experience of fall in 1-year } \\
\hline Yes & $2(10.53 \%)$ & $7(35 \%)$ & & $0.04^{*}$ & & \\
\hline No & $17(89.47 \%)$ & $13(65 \%)$ & & & & \\
\hline
\end{tabular}

${ }^{*} \mathrm{P}$-value is less than 0.05 , showed statistically significant difference in frequency compared by applying Chi square test

${ }^{\dagger}$ Not applicable, as the frequency in the ob

\section{The effect of lateral wedge insole}

The mean \pm SD use of LWI in KOA group was $8.30 \pm 1.49$ hours per day for four weeks. The mean \pm SD of individuals' expectation of beneficial effect of LWI was $4.35 \pm 0.67$. The mean \pm SD of perceived changes in pain and physical function by individuals was $4.45 \pm 0.75$, of which $18(85 \%)$ individuals categorized in the improvement group and $3(15 \%)$ in the no improvement group. The main outcome measure in this study was the immediate effect of LWI on general pain, and pain in walking compared to the self-reported pain in the last week of the study. By applying paired sample t-test in KOA at baseline, the significance effect of LWI on both pain scales were observed $(\mathrm{t}(19)=7.89 ; \mathrm{p}<0.001 ;$ and $\mathrm{t}(19)=8.72$; $\mathrm{p}<0.001$, respectively).

Moreover, the effect of LWI was assessed in KOA group at baseline and four-week follow-up. The pain and the time of TUG tests in all conditions were statistically reduced $(\mathrm{p}<0.001)$. The significant increases in times of OLB tests in standing on both limbs in three conditions were observed except for standing on left side in barefoot condition. Table 3 shows the details.

Table 3. Differences in symptomatic and functional changes within groups in individuals with knee osteoarthritis (KOA) at baseline and four-week follow-up

\begin{tabular}{|c|c|}
\hline Variables & $\begin{array}{l}\text { KOA at baseline-KOA at four-week follow-up } \\
\qquad \mathrm{t}(\mathrm{df}=19)\end{array}$ \\
\hline Average knee pain in the past week $(0-100)$ & $6.65\left(<0.001^{*}\right)$ \\
\hline Pain on walking (o-100) & $8.88\left(<0.001^{*}\right)$ \\
\hline Knee related $\mathrm{QOL}^{\dagger}(0-100)$ & $3.78\left(<0.001^{*}\right)$ \\
\hline Comfort with shoes with lateral wedge insole $(0-100)$ & $-2.10(0.05)$ \\
\hline $\mathrm{TUG}^{\ddagger}-\mathrm{WO}^{\mathrm{s}}(\mathrm{ms})$ & $3.70\left(<0.01^{*}\right)$ \\
\hline TUG-WS ${ }^{* *}(\mathrm{~ms})$ & $4.04\left(<0.001^{*}\right)$ \\
\hline TUG-WI ${ }^{++}(\mathrm{ms})$ & $3.76\left(<0.001^{*}\right)$ \\
\hline $\begin{array}{l}{ }^{*} \text { Denotes P-values }<0.05 \\
\text { Quality of Life } \\
{ }^{\ddagger} \text { Timed Up and Go Test } \\
{ }^{\mathrm{s}} \text { Barefoot } \\
{ }^{*} \text { With Shoes }\end{array}$ & \\
\hline
\end{tabular}


To consider the effect of foot conditions on TUG and OLB tests, repeated measurement ANOVA was applied. The foot conditions were enetered as the within subject variables, and the groups (CTL, KOA at baseline and follwoup) as the between subject factors. There was no significant effect of foot conditions on TUG test (F $(2,56)=3.69, \mathrm{p}=0.06)$ and also on interaction with groups $(F(4,56)=0.67, p=0.53)$. The conditions showed a significant effect on OLB of the right limb $(\mathrm{F}(2,56)=5.47$; $\mathrm{p}=0.01$ ). In pairwise comparisons, the LWI showed a significant difference with shoes condition $(p=0.02)$. But no significant effect of interaction with groups was observed $(\mathrm{F}(4,56)=1.51 ; \mathrm{p}=0.22)$. The effect of condition on OLB of the left limb was insignificant $(\mathrm{F}(2,56)=1.36$; $\mathrm{p}=0.26$ ), with a significant interaction with the effect of groups $(\mathrm{F}(4,56)=6.39 ; \mathrm{p}<0.001)$; of which $\mathrm{CTL}$ was statistically different from KOA groups at base-line and follow-up $(\mathrm{p}<0.001)$.

\section{Discussion}

The first aim of this study was to evaluate pain and risk of falls in early KOA. The immediate and four-week effect of LWI, as the conservative treatment, on early KOA was also evaluated. Higher score in pain and lower knee related QOL in individuals with KOA suggest that symptoms of KOA affected physical function and consequently QOL at the early stage of the disease. In addition, less ability to stand on one leg and more time to take TUG test showed that individuals with early KOA are at higher risk of falling. The immediate and four-week effect of LWI was observed on pain in individuals.

Pain and difficulties in physical activities in individuals with KOA create disability, social and economic burdens (26). Therefore, providing effective methods to reduce pain and improve daily activities in individuals with KOA is necessary. The observed improvement in pain and physical activities in early stage of the KOA in this study encourage the use of LWI as a conservative treatment. It has been reported that early defects in knee joint cartilage is reversible in younger people (15). Therefore, further investigation on the effect of LWI on clinical symptoms and knee joint structure is highly recommended.

In this study, a decrease in pain and an increase in quality of life were found as the results of the four-week use of the LWI in individuals with KOA. In a normally aligned knee, $60-70 \%$ of the loading is transferred through the medial compartment of the knee. In medial compartment KOA (27), this loading leads to a varus alignment of the knee (28). LWI leads to a more pronation foot position. This position encourages more upright position of the mechanical axis and decreases the distance of the mechanical axis and knee joint center of rotation. Therefore, a decrease in the loading of the medial compartment of the knee would be assumed (29). According to the result of this study, it can be assumed that LWI could lessen the pain in KOA individuals by decreasing load on the medial compartment and providing some shock absorbing. This assumption could be strengthened by the improvement shown in pain and TUG in KOA individuals after 4-week use of LWIs.
Further, LWI caused statistical improvement of physical activities in individuals with early KOA by a decreasing in time of TUG test and an increase in the ability of individuals to stand on one leg. Risk of falls is associated with pain and dysfunction in $\operatorname{KOA}(30,31)$, and pain is strongly associated with difficulty in activities of daily living (16). In our study, improvement of function and static standing balance test on one leg might be due to a decrease in pain and an increase in function. While pain and disability are the main reasons for people with $\mathrm{KOA}$ to seek for medical health services (17), the effect of conservative treatment, such as foot orthosis and shoe modifications, on pain and function at the early stage of the disease is important.

In our study, the mean daily use of LWI was 8.30 hours. According to the previous reports, the prevalence of adherence to wearing LWIs was 57 to $88 \%(12,32)$. Aggravation of foot pronation by LWI causes increased tension on the medial aspect of the foot, and leads to the individual's discomfort (33). In addition, it can cause local pressures on the sole of the foot (34). Adaptive insoles with longitudinal arch support may adjust foot alignment and decrease shock during walking (35). In this study, all individuals reported shoe comfort while they were wearing the insoles in their own shoes. Discomfort is a crucial factor to determine the compliance of individuals and adherence to a treatment. Therefore, to prevent over foot pronation, we embedded a mild medial longitudinal arch support in the insoles. The score of shoes comfort with LWIs was 88.00 at baseline, which was improved to 96.50 at follow-up in KOA group in this study. The result of the present study is in agreement with previous ones which reported the use of modified insoles could lessen discomfort and enhance clinical results (32).

The results of our study show the importance of the use of LWI as the conservative treatment at the early stage of the KOA. There were some limitations as well. First, the immediate and four-week effects were not assessed in a randomized controlled trial setting. Therefore, doubleblind randomized controlled trials to compare the immediate and long-term effect of LWI to neutral insole are required. Second, we considered four-week effect of LWI. The results of this study cannot be generalized to longterm effect of LWI.

\section{Conclusion}

KOA at early stage can affect the pain and quality of life. Use of $5^{\circ}$ LWI with shock absorbing feature and mild medial longitudinal arch support in routine shoes for 5 to 10 hours a day, can provide the potential for pain and function improvements in early KOA.

\section{Conflict of Interests}

The authors declare that they have no competing interests.

\section{References}

1. Alkan BM, Fidan F, Tosun A, Ardıçoğlu O. Quality of life and selfreported disability in patients with knee osteoarthritis. Mod Rheumatol. 2014;24(1):166-171. 
2. Jordan JM, Helmick CG, Renner JB, Luta G, Dragomir AD, Woodard $\mathrm{J}$, et al. Prevalence of hip symptoms and radiographic and symptomatic hip osteoarthritis in African Americans and Caucasians: The Johnston County osteoarthritis project. J Rheumatol. 2009;36(4):809-815.

3. Cross M, Smith E, Hoy D, Nolte S, Ackerman I, Fransen M, et al. The global burden of hip and knee osteoarthritis: estimates from the Global Burden of Disease 2010 study. Ann Rheum Dis. 2013.

4. Johnson VL, Hunter DJ. The epidemiology of osteoarthritis. Best Prac Res Clin Rheumatol. 2014;28(1):5-15.

5. Ndermann AM, Nigg BM, Humble RN, Stefanyshyn DJ. Orthotic comfort is related to kinematics, kinetics, and EMG in recreational runners. Med Sci Sports Exerc. 2003;195(9131/03):3510-1710.

6. Hallemans A, Ortibus E, Truijen S, Meire F. Development of independent locomotion in children with a severe visual impairment. Res Dev Disabil. 2011;32(6):2069-2074.

7. Messier SP, Loeser RF, Hoover JL, Semble EL, Wise CM. Osteoarthritis of the knee: effects on gait, strength, and flexibility. Arch Phys Med Rehabil. 1992;73(1):29-36.

8. Nguyen USDT, Felson DT, Niu J, White DK, Segal NA, Lewis CE, et al. The impact of knee instability with and without buckling on balance confidence, fear of falling and physical function: the Multicenter Osteoarthritis Study. Osteoarthritis Cartilage. 2014;22(4):527-534.

9. McAlindon TE, Bannuru RR, Sullivan MC, Arden NK, Berenbaum F, Bierma-Zeinstra SM, et al. OARSI guidelines for the non-surgical management of knee osteoarthritis. Osteoarthritis Cartilage. 2014;22(3):363-388.

10. Zhang W, Nuki G, Moskowitz RW, Abramson S, Altman RD, Arden NK, et al. OARSI recommendations for the management of hip and knee osteoarthritis: Part III: changes in evidence following systematic cumulative update of research published through January 2009. Osteoarthritis Cartilage. 2010;18(4):476-499.

11. Jones RK, Chapman GJ, Parkes MJ, Forsythe L, Felson DT. The effect of different types of insoles or shoe modifications on medial loading of the knee in persons with medial knee osteoarthritis: A randomised trial. J Orthop Res. 2015:n/a-n/a.

12. Demura SI, Yamada T, Shin S. Age and sex differences in various stepping movements of the elderly. Geriatr Gerontol Int. 2008;8(3):180-187.

13. Bennell KL, Bowles K-A, Payne C, Cicuttini F, Williamson E, Forbes A, et al. Lateral wedge insoles for medial knee osteoarthritis: 12 month randomised controlled trial. BMJ. 2011;342.

14. Parkes MJ, Maricar N, Lunt M, LaValley MP, Jones RK, Segal NA, et al. Lateral wedge insoles as a conservative treatment for pain in patients with medial knee osteoarthritis: a meta-analysis. JAMA. 2013;310(7):722-730.

15. Ding C, Jones G, Wluka AE, Cicuttini F. What can we learn about osteoarthritis by studying a healthy person against a person with early onset of disease? Curr Opin Rheumatol. 2010;22(5):520-527.

16. Felson DT, Niu J, Guermazi A, Sack B, Aliabadi P. Defining radiographic incidence and progression of knee osteoarthritis: suggested modifications of the Kellgren and Lawrence scale. Ann Rheum Dis. 2011;70(11):1884-1886.

17. Hunter DJ, Lo GH, Gale D, Grainger AJ, Guermazi A, Conaghan PG. The reliability of a new scoring system for knee osteoarthritis MRI and the validity of bone marrow lesion assessment: BLOKS (Boston-Leeds Osteoarthritis Knee Score). Ann Rheum Dis. 2008;67(2):206-211.

18. Kerrigan DC, Lelas JL, Goggins J, Merriman GJ, Kaplan RJ, Felson DT. Effectiveness of a lateral-wedge insole on knee varus torque in patients with knee osteoarthritis. Arch Phys Med Rehabil. 2002;83(7):889-893

19. Toda Y, Tsukimura N, Segal N. An optimal duration of daily wear for an insole with subtalar strapping in patients with varus deformity osteoarthritis of the knee. Osteoarthritis Cartilage. 2005;13(4):353360 .

20. Bellamy N, Kirwan J, Boers M, Brooks P, Strand V, Tugwell P, et al. Recommendations for a core set of outcome measures for future phase III clinical trials in knee, hip, and hand osteoarthritis. Consensus development at OMERACT III. J Rheumatol. 1997;24(4):799-802.

21. Salavati M, Mazaheri M, Negahban H, Sohani S, Ebrahimian M, Ebrahimi I, et al. Validation of a Persian-version of Knee injury and Osteoarthritis Outcome Score (KOOS) in Iranians with knee injuries.
Osteoarthritis Cartilage. 2008:16(10):1178-1182.

22. Bongue B, Dupré C, Beauchet O, Rossat A, Fantino B, Colvez A. A screening tool with five risk factors was developed for fall-risk prediction in community-dwelling elderly. J Clin Epidemiol. 2011;64(10):1152-1160.

23. Prince RL, Austin N, Devine A, Dick IM, Bruce D, Zhu K. EFfects of ergocalciferol added to calcium on the risk of falls in elderly highrisk women. Arch Intern Med. 2008;168(1):103-108.

24. Podsiadlo D, Richardson S. The timed" Up \& Go": a test of basic functional mobility for frail elderly persons. J Am Geriatr Soc. 1991;39(2):142-148.

25. Vellas BJ, Wayne SJ, Romero L, Baumgartner RN, Rubenstein LZ, Garry PJ. One-leg balance is an important predictor of injurious falls in older persons. J Am Geriatr Soc. 1997;45(6):735-738.

26. Robbins S, Gouw GJ, McClaran J. Shoe sole thickness and hardness influence balance in older men. J Am Geriatr Soc. 1992;40(11):10891094.

27. Menant JC, Steele JR, Menz HB, Munro BJ, Lord SR, Effects of footwear features on balance and stepping in older people. Gerontol. 2008;54(1): 18-23.

28. Menant JC, Steele JR, Menz HB, Munro BJ, Lord SR. Effects of walking surfaces and footwear on temporo-spatial gait parameters in young and older people. Gait Posture. 2009;29(3):392-397.

29. Vieira MF, Sacco IdCN, Nora FGdSA, Rosenbaum D, da Costa PHL. Footwear and foam surface alter gait initiation of typical subjects. PloS one. 2015;10(8):e0135821.

30. Foley SJ, Lord SR, Srikanth V, Cooley H, Jones G. Falls risk is associated with pain and dysfunction but not radiographic osteoarthritis in older adults: Tasmanian Older Adult Cohort study1. Osteoarthritis Cartilage. 2006;14(6):533-539.

31. Chester VL, Wrigley AT. The identification of age-related differences in kinetic gait parameters using principal component analysis. Clin Biomech. 2008;23(2):212-220.

32. Hsieh RL, Lee WC. Immediate and Medium-Term Effects of Custom-Moulded Insoles on Pain, Physical Function, Physical Activity, and Balance Control in Patients with Knee Osteoarthritis. J Rehabil Med. 2014;46(2):159-165.

33. Hinman RS, Bowles KA, Metcalf BB, Wrigley TV, Bennell KL. Lateral wedge insoles for medial knee osteoarthritis: Effects on lower limb frontal plane biomechanics. Clin Biomech. 2012;27(1):27-33.

34. Demura S, Shin S, Takahashi S, Yamaji S. Relationships between gait properties on soft surfaces, physical function, and fall risk for the elderly. Adv Aging Res. 2013;2(02):57.

35. Neogi T, Bowes MA, Niu J, Souza KM, Vincent GR, Goggins J, et al. Magnetic resonance imaging-based three $\square$ dimensional bone shape of the knee predicts onset of knee osteoarthritis: data from the Osteoarthritis Initiative. Arthritis Rheum. 2013;65(8):2048-2058. 